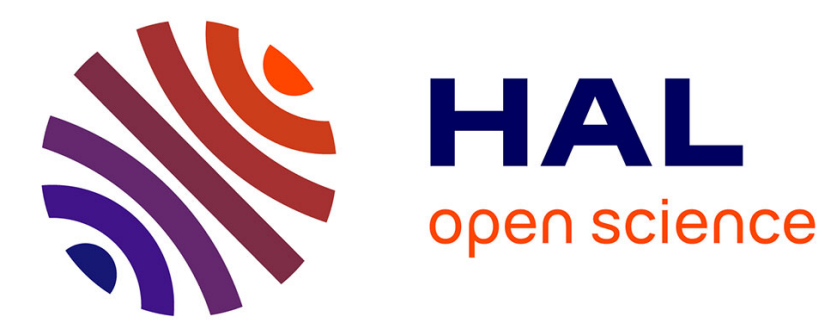

\title{
Robust sequence storage in bistable oscillators
}

David Colliaux, Pierre Bessière, Jacques Droulez

\section{To cite this version:}

David Colliaux, Pierre Bessière, Jacques Droulez. Robust sequence storage in bistable oscillators.

Nanoarch 2014, Jul 2014, France. 2 p. hal-01025504

\section{HAL Id: hal-01025504 \\ https://hal.science/hal-01025504}

Submitted on 18 Jul 2014

HAL is a multi-disciplinary open access archive for the deposit and dissemination of scientific research documents, whether they are published or not. The documents may come from teaching and research institutions in France or abroad, or from public or private research centers.
L'archive ouverte pluridisciplinaire HAL, est destinée au dépôt et à la diffusion de documents scientifiques de niveau recherche, publiés ou non, émanant des établissements d'enseignement et de recherche français ou étrangers, des laboratoires publics ou privés. 


\title{
Robust sequence storage in bistable oscillators
}

\author{
David Colliaux, Pierre Bessière and Jacques Droulez \\ ISIR, CNRS Université Pierre et Marie Curie, Paris \\ Email: surname.name@upmc.fr
}

\begin{abstract}
The versatility of nanodevices dynamics may allow original architectures for computation but care should be taken to handle fluctuations arising at this scale. In the network of oscillatory units which we propose, bistability with downquiescent and up-oscillatory states enable tolerance to noise in storage and retrieval dynamics for patterns or sequences. We illustrate this by simulations of the stochastic differential equations for a network with connectivity corresponding to stored patterns.
\end{abstract}

\section{INTRODUCTION}

Computational task like image segmentation or associative memory including sequences may be implemented through the dynamics of a network of coupled oscillators [1]. A major drawback limiting its use in real world applications is the integration of dynamics for nonlinear systems which are computationally demanding. Another concern about using oscillators for computational purpose is their low tolerance to noise impacting performance. We provide a solution for robust memory storage in a network of oscillators and propose that implementation relying on the dynamics of nanodevices, like magnetic tunnel junctions [2], would render oscillator-based computation amenable to real world applications.

\section{OSCILLATORY UP-STATE AND QUIESCENT DOWN-STATE}

\section{A. Dynamics of the bistable oscillator}

The network consist of units described by two coupled state variables $(\mathbf{s}, \phi)$ with the following dynamics:

$$
\begin{aligned}
\frac{d \mathbf{s}}{d t} & =-\mathbf{s}+w_{0} f(\mathbf{s})+\sigma\left(\cos \phi-I_{0}\right)+I_{e x t}+d \boldsymbol{\xi}_{t} \\
\frac{d \boldsymbol{\phi}}{d t} & =\omega+(\beta-\rho \mathbf{s}) \sin \boldsymbol{\phi}
\end{aligned}
$$

Apart from linear relaxation, the dynamics for $\mathbf{s}$ includes three nonlinear inputs: self-feedback modulated by $w_{0}$ where $f$ is a smooth approximation of the Heaviside-step function thresholded at 0.5 , feedback from the oscillatory variable and external inputs from stimulation and/or other units in the network. The parameters are listed in Table I and the particular values in forthcoming figures were guided by previous studies in a deterministic framework [1]. The model we consider here includes stochastic perturbation by independent Gaussian processes $\boldsymbol{\xi}_{t}$ with 0 mean, variance 1 and no temporal correlation. The constant input to the $\mathbf{s}$ variable $I_{0}$ is thus chosen so that units converge to the stable fixed point $\left(0, \phi_{0}\right)$ in the deterministic case. The stochastic dynamics were integrated using a strong order 1 Taylor scheme [3].

A first case is illustrated in Figure 1 with a single unit, $\mathbf{s}=(s)$ and $\phi=(\phi)$, for 3 values of nonlinear feedback.
TABLE I. PARAMETER VALUES USED IN THE SIMULATIONS

\begin{tabular}{c||c} 
Parameter name & Parameter value \\
\hline$\beta:$ self-coupling of $\phi$ & 1.2 \\
$\omega:$ intrinsic frequency & 1. \\
$\rho:$ s to $\phi$ coupling & .9 \\
$\sigma: \phi$ to s coupling & .9 \\
$d:$ noise intensity $(2$. std $)$ & $.1($ varied $)$ \\
$w_{0}:$ self-coupling of $\mathbf{s}$ & $1($ varied $)$ \\
$f(s):$ soft thresholding function & $\frac{\tanh (10(s-0.5))-1}{2}$ \\
$I_{0}:$ pulse strength & 1 \\
$N:$ Number of units & 225 \\
$K:$ number of active units in a pattern & 30
\end{tabular}

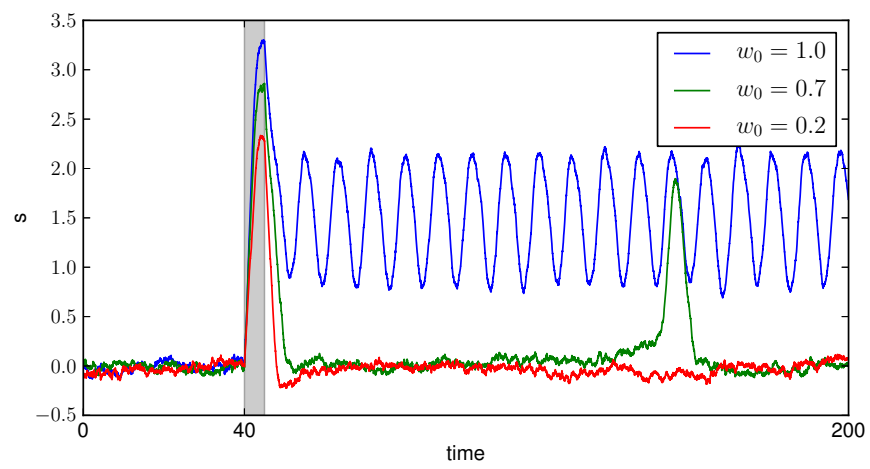

Fig. 1. Time course of $s$ for a single unit at 3 values of feedback gain $w_{0}$. Transition to oscillatory up state after transient stimulation with a short pulse occurs when feedback is strong enough. The shaded area denotes the time interval during which stimulation is applied.

After transient stimulus, $I_{e x t}=I_{0}$, is applied the system is confined close to the up-state limit cycle of the deterministic system if feedback is strong enough whereas it stays close to the resting state for weak feedback. As shown by the trajectory at $w_{0}=0.7$, some noise driven excursion close to the up-state are possible but those are not frequent and transient.

\section{B. Coupling for an associative memory}

To implement associative memory in a network of $N$ units, we consider 3 binary patterns $\left(\mathbf{p}^{(\mathbf{1})}, \mathbf{p}^{(\mathbf{2})}, \mathbf{p}^{(\mathbf{3})}\right)$ with $N$ entries, $K$ being non-zero $(K=O(\sqrt{N})$ ), [and nonoverlapping across patterns for simplicity]. Maintenance or recall of a pattern is encoded in the activity $s_{i}$ corresponding to non-zero entries of the pattern. Each unit receive inputs from external stimuli when it is presented and from other units of the network, for a unit of index i:

$$
I_{i}^{e x t}=p_{i}^{(k)}(t)+\sum_{j} w_{j i} f\left(s_{j}\right) .
$$




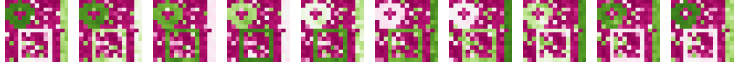

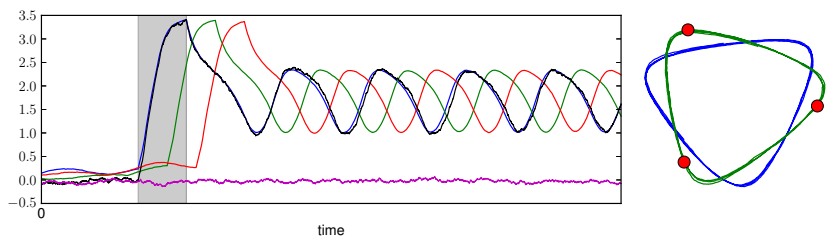

Fig. 2. Sequence maintenance: (UP) Snapshots of activity in the network over a sequence cycle. (DOWN-LEFT) Dynamics for the ovelaps. Patterns are triggered sequentially via stimulation pulses of the full pattern. (DOWNRIGHT) Sequence trajectory in reduced space.

A pattern $\mathbf{p}$ is stored by connecting units according to their co-occurence in stored patterns:

$$
w_{i j}=\frac{1}{K} \sum_{k \in(1,2,3)} p_{i}^{(k)} p_{j}^{(k)} .
$$

This type of symmetric connections are commonly used in neuronal network [4] were memory retrieval is achieved through a fixed point. The same type of dynamics are achieved when both $\rho$ and $\sigma$ are 0 but for the parameters listed in Table I the memory of the pattern is maintained via a limit cycle.

\section{SEQUenCE StORAGE AND ROBUStNESS ASSESMENT}

\section{A. The sequence trajectory}

To account for the retrieval of a pattern, it is useful to monitor the overlap between the activity of the network and the stored patterns:

$$
o_{t}^{(k)}=\mathbf{s}_{t} \cdot \mathbf{p}^{(\mathbf{k})} .
$$

Morever, when patterns are stimulated sequentially, the dynamics of the network activity cycle with overlaps having phase difference corresponding to the differences in timing when pulse inputs were triggered.

The dynamics of the network can thus be mapped to the plane by restricting the trajectories to the 2 first principal components and different sequences will have different trajectories in this plane. This is illustrated in Figure 2-(DOWN-RIGHT) when storing $\mathbf{p}_{\mathbf{1}} \rightarrow \mathbf{p}_{\mathbf{2}} \rightarrow \mathbf{p}_{\mathbf{3}}$ (green) and $\mathbf{p}_{\mathbf{1}} \rightarrow \mathbf{p}_{\mathbf{3}} \rightarrow \mathbf{p}_{\mathbf{2}}$ (blue). The two trajectories are separable as their cycle have different orientations.

\section{B. Robustness of the storage}

To investigate the separability of the stored patterns, we considered the correlation between the normalized overlaps, which is negative when patterns can be well separated. We compute the average separability across pattern pairs for various intensity of the noise:

$$
S \propto \sum_{k \neq l} \sum_{t} \bar{o}_{t}^{(k)} \cdot \bar{o}_{t}^{(l)} .
$$

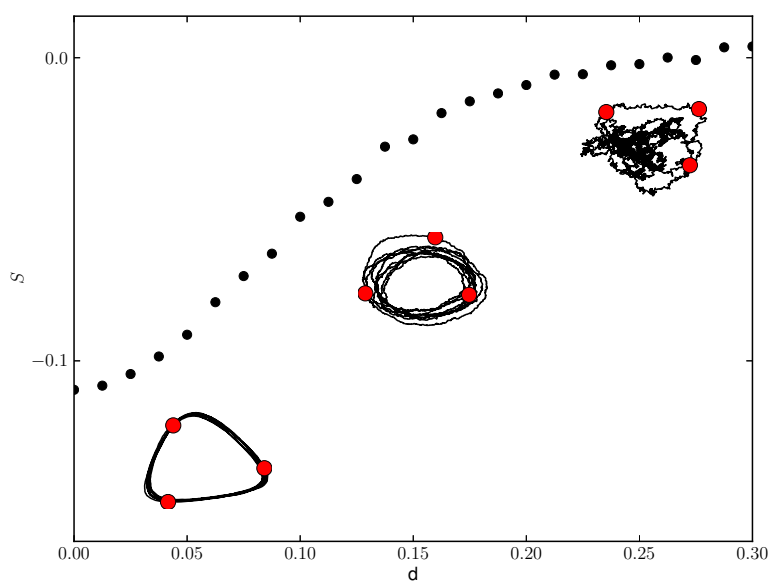

Fig. 3. Robustness of stored patterns: separability $S$ of the patterns for various noise intensities. Trajectory in reduced space for low, intermediate and high noise intensities are also depicted as insets.

In Figure 3, as separability degrades under increasing noise strength, we compare the sequence trajectories. For weak noise, the patterns are separable and their stimulation order can be determined. For intermediate noise, the patterns may be decoded but their stimulation order is lost because the cycle has no specific orientation. Finally for strong noise intensity, patterns cannnot be distinguished at all.

\section{CONCLUSION}

We demonstrated the storage of a sequence in the dynamics of a network of bistable oscillators and presented analysis tool to asses its robustesnes under noisy input. If implemented with magnetic tunnel junctions, the model we propose would provide an efficient solution to the robust storage of sequences.

More sophisticated scenarios like retrival of a sequence from presentation of the first element could be studied and additional mechanism (like winner-take-all via global inhibition) could be considered to enhance robustness.

\section{ACKNOWLEDGMENT}

This work was supported by the european project FP7 BAMBI.

\section{REFERENCES}

[1] D. Colliaux, C. Molter, and Y. Yamaguchi, "Working memory dynamics and spontaneous activity in a flip-flop oscillations network model with a milnor attractor," Cognitive Neurodynamics, vol. 3, no. 2, pp. 141-151, Jun. 2009. [Online]. Available: http://link.springer.com/10. 1007/s11571-009-9078-0

[2] N. Locatelli, V. Cros, and J. Grollier, "Spin-torque building blocks," Nat Mater, vol. 13, no. 1, pp. 11-20, Jan. 2014. [Online]. Available: http://dx.doi.org/10.1038/nmat3823

[3] P. E. Kloeden, Peter E., Numerical Solution of Stochastic Differential Equations. Springer, 1992.

[4] J. J. Hopfield, "Neural networks and physical systems with emergent collective computational abilities," Proceedings of the National Academy of Sciences, vol. 79, no. 8, pp. 2554-2558, Apr. 1982. [Online]. Available: http://www.pnas.org/content/79/8/2554.abstract 